\title{
Ecological Momentary Assessment of
}

\section{Non-Menstrual Pelvic Pain: Potential Pathways of Central Sensitization in Adolescents and Young Adults with and without Primary Dysmenorrhea}

This article was published in the following Dove Press journal:

Journal of Pain Research

\section{Laura C Seidman (D) Catherine R Temme' Lonnie K Zeltzer ${ }^{2}$ Andrea J Rapkin ${ }^{3}$ \\ Bruce D Naliboff ${ }^{4}$ Laura A Payne ${ }^{1,5}$}

'Division of Women's Mental Health, McLean Hospital, Belmont, MA, USA; ${ }^{2}$ Department of Pediatrics, David Geffen School of Medicine at UCLA, Los Angeles, CA, USA; ${ }^{3}$ Department of Obstetrics and Gynecology, David Geffen School of Medicine at UCLA, Los Angeles, CA, USA; ${ }^{4}$ Oppenheimer Center for Neurobiology of Stress and Resilience, David Geffen School of Medicine at UCLA, Los Angeles, CA, USA; ${ }^{5}$ Department of Psychiatry, Harvard Medical School, Boston, MA, USA
Correspondence: Laura C Seidman McLean Hospital, Mail Stop 306, II 5 Mill Street, Belmont, MA 02478, USA $\mathrm{Tel}+$ I 6178552746

Email LCSeidman@mclean.harvard.edu
Purpose: Primary dysmenorrhea (PD; menstrual pain without an identified organic cause) has been proposed as a possible risk factor for the development of chronic pelvic pain, but the mechanism through which this process occurs is unknown. One possible mechanism is central sensitization - alterations in the central nervous system that increase responsiveness to pain leading to hypersensitivity. Repeated episodes of pain, such as those experienced over time with PD, may alter how the brain processes pain. Ecological momentary assessment (EMA; collection of data in real time in participants' natural environments) is a novel data collection method that may help elucidate pain occurring during non-menstrual cycle phases. Patients and Methods: The current observational study assessed the feasibility and acceptability of using EMA via text messages to collect pelvic pain data during menstrual and non-menstrual cycle phases in a community sample of adolescents and young adults (AYA) aged 16-24 years with and without PD and explored occurrence rates and intensity of non-menstrual pelvic pain (NMPP) in each of these groups.

Results: Thirty-nine AYA with PD and 53 healthy controls reported pelvic pain level via nightly text message. Global response rate was $98.5 \%$, and all participants reported that the EMA protocol was acceptable. AYA with PD reported higher intensity (2.0 vs 1.6 on $0-10$ numeric rating scale; $\mathrm{p}=0.003$ ) and frequency ( $8.7 \%$ vs $3.1 \%$ of days; $\mathrm{p}=0.004)$ of NMPP compared to healthy controls. Conclusion: The EMA protocol was feasible and acceptable. Though both the intensity and frequency of NMPP were low and at levels that would not typically warrant clinical assessment or intervention, these repeated nociceptive events may represent a potential mechanism contributing to the transition from cyclical to chronic pelvic pain in some individuals.

Keywords: ecological momentary assessment, primary dysmenorrhea, menstrual pain, adolescents and young adults, central sensitization

\section{Introduction}

Primary dysmenorrhea (PD), defined as menstrual pain without an identified organic cause, is estimated to affect between $45 \%$ and $95 \%$ of menstruating girls and women and is a leading cause of work- and school-related absences. ${ }^{1}$ Recent studies have established PD as a potential risk factor for the development of chronic pelvic pain, 2,3 however, the mechanism through which this process occurs is unknown.

One proposed mechanism for the transition from cyclical to chronic pelvic pain is central sensitization (CS). $\mathrm{CS}$ is characterized by alterations in the central 
nervous system that increase responsiveness to pain leading to hypersensitivity. ${ }^{4,5} \mathrm{CS}$ has been shown to play a role in numerous chronic pain conditions, including chronic pelvic pain. ${ }^{6-8}$ Specifically, repeated experiences of nociceptive input from the reproductive organs and viscera, as is the case with PD, can result in neuronal hypersensitivity and hyperalgesia and can change how the brain processes pain-related information. ${ }^{4,5}$ Brain imaging studies have shown altered brain structure and function in women with PD compared to controls, even during non-painful cycle phases. ${ }^{10-12}$ And experimental pain paradigms have shown evidence of $\mathrm{CS}$ in women with $\mathrm{PD}$ as demonstrated by increased pain sensitivity to laboratory stimuli compared to controls. $^{13-15}$ In light of these differences, which appear to be stable across menstrual cycle phases, ${ }^{13-15}$ studying the years between the onset of painful menstruation and the development of chronic pelvic pain could be critical to understanding this transition. One study found that the average time between the emergence of dysmenorrhea and consultation with a doctor for dysmenorrhea was 5.1 years. ${ }^{2}$ Assessing pain during this time when symptoms are not otherwise recorded or reported may enable identification of risk factors for the development of chronic pain.

Novel methods, such as Ecological Momentary Assessment (EMA), may be critical for determining the presence and severity of symptoms outside of the menstrual phase. EMA is a procedure for the collection of data in real time in participants' natural environments ${ }^{16}$ that has evolved from paper diaries to advanced methods utilizing cell phones and ambulatory monitoring devices. ${ }^{17}$ A benefit of electronic EMA is the reduction in recall biases commonly seen in paper diaries due to back-filling and distortion of emotional memories. ${ }^{18}$

EMA has been used to assess pain intensity and related symptoms in a variety of chronic pain conditions, ${ }^{17,19}$ but EMA studies to assess menstrual or pelvic pain data across the menstrual cycle in women with PD are lacking. A number of unique features of using EMA for assessment of menstrual pain support the need to establish its feasibility and acceptability in this population. First, the nature of pain that occurs on an approximately monthly basis inherently requires longer monitoring than typical EMA for chronic pain. ${ }^{17,19}$ Furthermore, the experience of PD in an otherwise-healthy population varies from chronic pain in that it is cyclical, predictable, and occurs on a relatively lower percentage of days.

The aims of this study were therefore to conduct secondary data analysis in an existing data set to: 1) assess the feasibility and acceptability of using EMA via end-of-day (EOD) text messages to collect pelvic pain data during menstrual and non-menstrual cycle phases in a community sample of adolescents and young adults (AYA) with and without PD; and 2) explore occurrence rates and intensity of non-menstrual pelvic pain (NMPP) in each of these groups.

\section{Methods \\ Participants}

Participants included 92 female AYA ages 16-24 years (mean $=20.8 ; \mathrm{SD}=2.0)$. Participants were categorized as having primary dysmenorrhea (PD group; $n=39$ ) if they reported an average menstrual pain rating of " 4 " or higher on a 0 (no pain) to 10 (worst pain possible) numeric rating scale (NRS; see Measures below). Control participants (control group; $n=53$ ) rated average menstrual pain as "3" or below on the same scale. ${ }^{14,20}$ Demographic and menstrual history characteristics of the two groups and the overall sample are presented in Table 1. Apart from average menstrual pain rating, there were no differences between groups on any measure except that a larger percent of the PD group was Hispanic or Latino (see Seidman et $\mathrm{al}^{21}$ for discussion). Participants were enrolled in a larger study examining experimental pain across the menstrual cycle, which found enhanced pain sensitivity at all phases of the menstrual cycle in AYA with PD compared to healthy controls, but no evidence of enhanced excitatory or deficient inhibitory mechanisms. ${ }^{14}$ Results presented in this manuscript were exploratory analyses from, and represent the full sample of, the larger study.

Three hundred and fifty-three individuals were screened for eligibility by telephone. Of those, nine $(2.5 \%)$ were no longer interested in participating and 245 (69.4\%) were excluded. Reasons for exclusion included: use of hormonal contraceptives or other exogenous hormones in the prior 3 months $(n=117)$; irregular, long, or short cycle lengths $(n=25)$; inconsistent patterns of menstrual pain $(n=25)$; participants representing cells that were already at maximum enrollment (age, $n=6$; PD group, $n=19$ ); and other exclusionary criteria ( $n=6$; see Figure 1$)$. An additional 47 participants were excluded due to selectively enrolling those with lower and higher levels of average menstrual pain in order to create more distinct groups. Ninety-nine individuals were invited to participate, of whom seven declined as no longer being interested or available. See Figure 1 for a study enrollment flow diagram. 
Table I Demographic and Menstrual History Characteristics

\begin{tabular}{|c|c|c|c|c|}
\hline & Control Group $(n=53)$ & PD Group $(n=39)$ & $P$ value & Total $(n=92)$ \\
\hline Age (years) & $20.7(1.8)$ & $21.1(2.1)$ & ns & $20.8(2.0)$ \\
\hline Ethnicity & & & 0.011 & \\
\hline Hispanic/Latino & $8(15.1)$ & $15(38.5)$ & & $23(25.0)$ \\
\hline Non-Hispanic/Non-Latino & $45(84.9)$ & $24(61.5)$ & & $69(75.0)$ \\
\hline Race & & & ns & \\
\hline White & $21(39.6)$ & $20(51.3)$ & & $41(44.6)$ \\
\hline Black/African-American & $3(5.7)$ & $5(12.8)$ & & $8(8.7)$ \\
\hline Asian & $28(52.8)$ & 14 (35.9) & & $42(45.7)$ \\
\hline American Indian/Alaska Native & I (I.9) & $0(0.0)$ & & I (I.I) \\
\hline Average menstrual pain rating & $0.7(0.8)$ & $7.1(1.7)$ & 0.000 & $3.4(3.4)$ \\
\hline Age at menarche (years) & $12.2(1.4)$ & $11.9(1.2)$ & ns & 12.1 (I.3) \\
\hline
\end{tabular}

Note: Data presented as mean (SD) for continuous variables and $\mathrm{N}$ (\% of group) for categorical variables.

Abbreviations: PD, primary dysmenorrhea; ns, not significant.

\section{Procedures}

Details about recruitment, enrollment, and the study protocol have been extensively described elsewhere ( see $^{14,21}$ ). In brief, participants were recruited from emails to university students, online postings, word of mouth referrals, and participants from previous studies. Participants were screened by phone to assess whether the potential participant met any of the following exclusion criteria: 1) illness or injury that would potentially impact pain task performance (eg, fever and flu symptoms) or that would affect sensitivity of the extremities (eg, Raynaud disease); 2) daily use of opioids at the time of study participation; 3 ) developmental delay, diagnosis of autism, or significant cognitive impairment that may preclude understanding of study procedures; 4) use of hormonal contraceptives in the previous 3 months; 5$)$ irregular menstrual cycles $(<24$ or $>32$ days); or 6) currently pregnant. Written informed consent was obtained in person from young adult participants, and written teen assent and parent permission were obtained from minor participants and a legal guardian. Data collection occurred from October 2014 to June 2016. The study was approved by the Institutional Review Board at the University of California, Los Angeles (Protocol \# 13-000637) and was conducted in accordance with the Declaration of Helsinki. Each participant earned up to $\$ 250$ cash for participation in the larger study.

\section{EMA: Nightly Text Messages}

Participants were sent an automatic nightly text message between $8 \mathrm{pm}$ and midnight every night. Participants were able to self-select the time within that window at which they preferred to receive the text messages. Text messages inquired about whether or not the participant was menstruating (yes/no) and her level of pelvic pain that day, regardless of menstruation status, on the 0-10 NRS. Participants were trained on text message completion during the intake visit, which included providing them with information about the possibility to experience pelvic pain when not on your period; the research coordinator ensured that the participant understood this distinction (ie, having pelvic pain while not on your period) and how she should reply to the text messages each day. The study coordinator followed-up the following day with any participant who had not responded to the previous night's text message.

Three participants became non-responsive to the nightly text messages and were not reachable by phone or email. Study protocol outlined that these participants were classified as withdrawn from the study if they did not respond to three phone calls and/or emails and also did not respond to nightly text messages for 2 weeks. Thus, 2 weeks' (14 days') worth of unanswered text messages are included for these three participants. Similarly, one participant stopped responding to text messages but contacted the study team to report that she had an illness and would not be able to come in for her visit. Automatic text messages were stopped for this participant and the 1 week (7 days) of missing data at the end of her study participation were kept as missing data. Lastly, one participant who had already completed all of her in-person visits stopped responding to the nightly text messages starting the fourth day of her subsequent period. Four days of missing text data were counted at the end of her participation to 


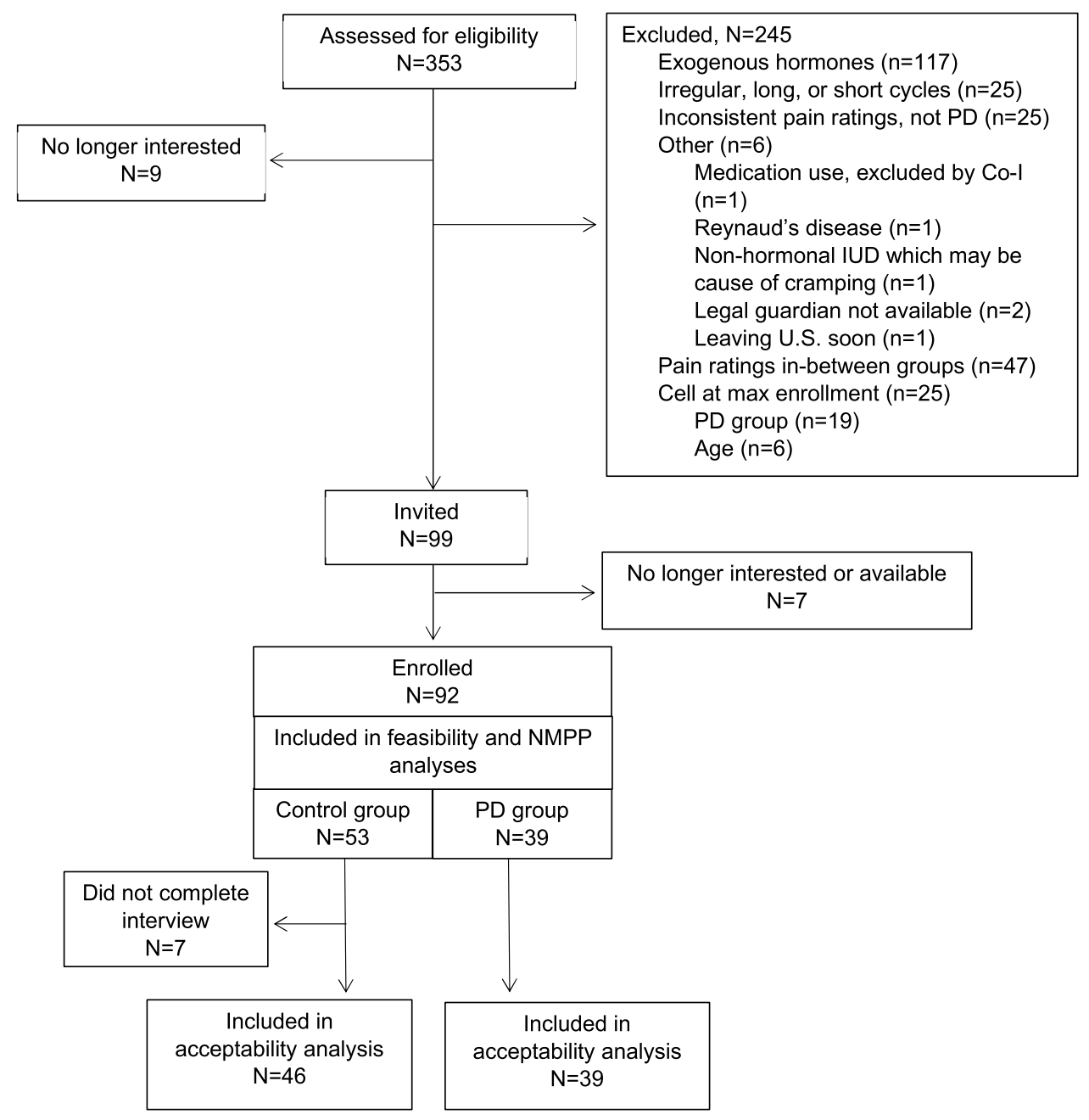

Figure I Study enrollment flow diagram.

account for the likely last 3 days of her period (based on the observed length of her previous period as 6 days) and 1 day afterwards (included for all participants as a way to inform study coordinator to stop the automatic texts).

\section{Interviews}

Semi-structured interviews were conducted with all participants after their final laboratory session. Interviews were conducted in person in a private room within a suite of clinical research offices and labs. Of the 92 enrolled participants, 7 did not complete interviews due to: 1) dropping out of the study before completing any experimental study visits $(\mathrm{n}=5)$ or 2 ) anovulation precluding the conducting of subsequent ovulatory and luteal phase lab sessions during which the interview would have occurred $(n=2)$. Interview audio was transcribed by a professional transcription company and reviewed by research study personnel for completeness and accuracy. Three participants declined to be audio recorded so the interviewer took notes and typed them immediately following the interview. Interview guides contained two questions regarding text messages: "How did the automatic text messaging work for you?" [Question 1] and "Is there any way of communicating that you think would have worked better?" [Question 2]. Responses to these two questions, as well as mentions of text messaging elsewhere throughout the interview, were compiled and reviewed.

\section{Measures}

\section{Demographics and Menstrual History}

A measure designed for this study was used to collect participant demographic and menstrual history data, including age, race, ethnicity, and age at menarche.

\section{Response Rate}

Individual response rate was calculated by dividing the number of answered text messages by the number of text 
messages sent for each participant. Global response rate was calculated by dividing the overall number of text messages answered (across all participants) by the overall number of text messages sent. Feasibility was determined by global response rate, such that the procedure would be determined to be feasible if the global response rate was at least $80 \%{ }^{22,23}$ The protocol would be determined to be acceptable if $80 \%$ of the participants confirmed that the text messages worked well for them and that there was not a better way of communicating.

\section{Numeric Rating Scale}

During the telephone screening, participants rated their average menstrual pain on a 0 (no pain) to 10 (worst pain possible) NRS. The NRS is a valid and reliable measurement of pain and is widely used throughout the literature, ${ }^{24,25}$ including with adolescent populations. ${ }^{26,27}$ The NRS was also used to rate daily pelvic pain.

\section{Non-Menstrual Pelvic Pain - Intensity (NMPP-I)}

Two variables were calculated to assess the intensity of the pelvic pain reported across days during which participants were not menstruating. The first, NMPP-Ip, is the mean NRS value for all painful non-bleeding days (ie, NRS $>0$, when they had pelvic pain, how intense was it?) The second, NMPP-Ia, is the mean NRS value for all nonbleeding days (ie, what is their overall intensity of pelvic pain across all non-bleeding days?). NMPP-Ip and NMPPIa were calculated based on the overall data set of days (ie, each day is weighted equally in the calculations because they are independent of participant).

\section{Non-Menstrual Pelvic Pain - Occurrence (NMPP-\%)}

The percentage of days during which the participant was not menstruating that she reported experiencing any level of pelvic pain (ie, $>0$ on the NRS).

\section{Statistical Analyses}

Sample size for the larger study was determined by power analysis; no post hoc power analyses were conducted for the current investigation..$^{28,29}$ Descriptive statistics were used to assess feasibility and acceptability. Differences between the PD and control groups were compared using Pearson Chi-Square tests for categorical variables, independent sample $t$-tests for normally distributed continuous variables (age), and Independent Samples Mann-Whitney $U$-Tests for variables that Shapiro-Wilk Tests of Normality determined to be not normally distributed (average menstrual pain rating, age at menarche, length of participation, response rate, NMPP-Ip, NMPP-Ia, and NMPP-\%). Correlations were conducted using Kendall's Tau-b correlations.

\section{Results \\ Response Rate}

Average length of study participation was 78.3 days $(\mathrm{SD}=35.5 ; \min =22 ; \max =171)$. Length of participation in the broader study was determined by a number of factors, including participant's menstrual cycle phase at intake and which phase she was randomized to start lab visits; presumptive anovulatory cycles delaying study visits; scheduling issues related to illness, holidays, and extended school breaks, etc. There was no significant difference in length of study participation between groups.

Ninety participants $(97.8 \%$ of sample) agreed to receive daily text messages. One participant did not have text messages included in her phone plan and the other was an international student for whom daily texts would have been too expensive. These participants were sent automatic nightly emails. An additional four participants were unable to receive text messages for a portion of the study due to international travel or transitioning between phones or plans. These participants were switched to nightly emails for the relevant period of time and switched back to text messages as they were able. References throughout the manuscript to text messages and responses include automatic emails and responses to those emails. Text messages were paused for two participants over extended school breaks during which they were out of town (1 month and 2.5 months, respectively) and resumed a few weeks prior to each participant's return to campus.

Global response rate was very high $-98.5 \%$ of all text messages (7091 of 7199) were answered. Individual response rates ranged from $72.6 \%$ to $100 \%$, and 71 participants (77.2\% of the sample) had a response rate of $100 \%$. Response rate did not differ between groups (control group: $\mathrm{M}=97.7, \mathrm{SD}=6.2$; $\mathrm{PD}$ group: $\mathrm{M}=99.4, \mathrm{SD}=1.6$ ). The three lowest completion rates were from participants with 2 weeks' of missing data prior to removal from the study, as described above. Excluding the missing data at the end of participation for those three individuals, the global response rate was $99.1 \%$ and individual response rates ranged from $89.1 \%$ to $100 \%$. See Table 2 for group differences in text message data and Table 3 for a complete breakdown of days. 
Table 2 Group Differences in Text Message and NMPP Data

\begin{tabular}{|c|c|c|c|c|}
\hline & Control Group $(n=53)$ & PD Group $(n=39)$ & $P$ value & $\begin{array}{l}\text { Total } \\
(n=92)\end{array}$ \\
\hline Length of participation (days) & $84.8(38.7)$ & $69.3(28.7)$ & ns & $78.3(35.5)$ \\
\hline Min & 22 & 25 & & 22 \\
\hline $\operatorname{Max}$ & 171 & 143 & & $|7|$ \\
\hline Median & 80 & 66 & & 70 \\
\hline Response rate (\%) & $97.7(6.2)$ & $99.4(1.6)$ & ns & $98.4(4.9)$ \\
\hline Min & 72.6 & 91.7 & & 72.6 \\
\hline Max & 100.0 & 100.0 & & 100.0 \\
\hline Median & 100.0 & 100.0 & & 100.0 \\
\hline NMPP-IP & $1.60(1.0)$ & $2.00(1.3)$ & 0.003 & $1.87(1.2)$ \\
\hline NMPP-la & $0.04(0.29)$ & $0.17(0.66)$ & 0.000 & $0.09(0.47)$ \\
\hline NMPP-\% & $3.1(5.2)$ & $8.7(10.7)$ & 0.004 & $5.5(8.4)$ \\
\hline Min & 0.0 & 0.0 & & 0.0 \\
\hline $\operatorname{Max}$ & 27.3 & 40.7 & & 40.7 \\
\hline Median & 1.2 & 4.3 & & 2.5 \\
\hline
\end{tabular}

Abbreviations: PD, primary dysmenorrhea; NMPP, non-menstrual pelvic pain; Ip, mean NRS value for all painful non-bleeding days; la, mean NRS value for all non-bleeding days; NMPP-\%, percentage of days during which the participant was not menstruating that she reported experiencing any level of pelvic pain.

Table 3 Breakdown of Numbers of Total Days and Non-Bleeding Days During Which Participants Received and Responded to Text Messages

\begin{tabular}{|c|c|c|c|}
\hline & $\begin{array}{l}\text { Control Group } \\
(n=53)\end{array}$ & $\begin{array}{l}\text { PD Group } \\
(n=39)\end{array}$ & $\begin{array}{l}\text { Total } \\
(n=92)\end{array}$ \\
\hline Total no. of days participants enrolled and receiving text message & 4496 & 2703 & 7199 \\
\hline No. of days answered & 4408 & 2683 & 7091 \\
\hline No. of days un-answered & 88 & 20 & 108 \\
\hline No. of non-bleeding days that a text was answered & 3581 & 2123 & 5704 \\
\hline No. of answered non-bleeding days with NRS $=0$ & 3493 & 1946 & 5439 \\
\hline No. of answered non-bleeding days with NRS $>0$ & 88 & 177 & 265 \\
\hline \multicolumn{4}{|l|}{$N(\%)$ of days that were rated } \\
\hline $\mathrm{I}-3$ & $84(95.5)$ & $161(91.0)$ & $245(92.5)$ \\
\hline $4-6$ & $3(3.4)$ & $14(7.9)$ & $17(6.4)$ \\
\hline $7-10$ & I (I.I) & $2(I . I)$ & $3(I . I)$ \\
\hline
\end{tabular}

Abbreviations: PD, primary dysmenorrhea; NRS, numeric rating scale.

\section{Acceptability}

Review of the qualitative interview data revealed that $100 \%$ of the participants who completed nightly text messages found the protocol acceptable, which exceeds the minimum of $80 \%$ required to meet the acceptability criterion. Sample quotations demonstrate different aspects of EMA via text message. In response to Question 1 ("How did the automatic text messaging work for you?"), sample quotations include: "It was totally fine, it was really easy."; "It was actually good because 10 o'clock is around the time when I'm usually on my phone a lot, so it was the right timing."; "It was good because in the night, if I didn't get to it right away, I would get to it eventually."; "It was pretty easy just because I'm on my phone all the time."; "When I received them I would complete them immediately." In response to Question 2 ("Is there any way of communicating that you think would have worked better?"), "No, text message is fast and convenient."; "No. I think nowadays texting is the best way to reach, especially someone like me, I'm always on the move. It's the best thing and the quickest way."; "No, that was perfect cause it is the first thing I check." 


\section{Non-Menstrual Pelvic Pain - Intensity on Painful Days (NMPP-IP)}

Of all the non-bleeding days that were rated as painful (ie, NRS $>0 ; n=265$ ), the vast majority (92.5\%) were rated between 1 and 3 on the NRS (see Table 3). The average NRS rating across painful non-menstrual days (ie, NRS $>0$ ) was $1.87(\mathrm{SD}=1.2)$ across the whole sample, and was significantly higher in the PD group $(\mathrm{M}=2.00, \mathrm{SD}=1.3) \mathrm{com}$ pared to the control group $(\mathrm{M}=1.60, \mathrm{SD}=1.0 ; \mathrm{p}=0.003)$.

\section{Non-Menstrual Pelvic Pain - Intensity on All Days (NMPP-la)}

The average NRS rating across all non-menstrual days (ie, $\mathrm{NRS} \geq 0)$ was $0.09(\mathrm{SD}=0.47)$ across the whole sample, and was also significantly higher in the PD group $(\mathrm{M}=0.17$, $\mathrm{SD}=0.66)$ compared to the control group $(\mathrm{M}=0.04$, $\mathrm{SD}=0.29 ; \mathrm{p}=0.000$ ).

\section{Non-Menstrual Pelvic Pain - Percent of Days Experienced as Painful (NMPP-\%)} NMPP ranged from $0 \%$ to $40.7 \%$ of days $(\mathrm{M}=8.7$, $\mathrm{SD}=10.7)$ in the $\mathrm{PD}$ group and from $0 \%$ to $27.3 \%$ of days $(\mathrm{M}=3.1, \mathrm{SD}=5.2)$ in the control group. Average NMPP-\% in the PD group was significantly higher than in the control group ( $\mathrm{p}=0.004$, see Figures 2 and 3 for histograms of NMPP distributions, and Table 2 for group differences in NMPP). NMPP-\% was not correlated with age $(\tau \mathrm{b}=0.07, \mathrm{p}=0.36)$ or age at menarche $(\tau \mathrm{b}=-.05$, $\mathrm{p}=0.55)$. See Figure 4 for a sample pictorial representation of low-level pelvic pain across the menstrual cycle.

\section{Discussion}

Our data show that EMA via EOD text messages is a feasible and acceptable method of menstrual and pelvic pain data collection in female AYA. While completion rate in the EMA literature is not always reported and can vary widely depending on demographics, data collection modality, and number of prompts per day, ${ }^{30}$ the $98.5 \%$ global response rate in the current study is on the very high end of those who report response rate. ${ }^{17,19,31}$ Others have noted that having the technology tailored to the adolescent population and providing a tutorial on how to use the technology effectively can help increase response rates, ${ }^{32}$ both of which were done in the current study. Cell phones have had an increasingly important role in our society for all age groups, including adolescents, ${ }^{32-34}$ and the vast majority of young adults report using cell phones for texting. ${ }^{35}$ EMA using cell phones is also more convenient, because most people are already carrying their cell phone around with them, which can help increase response rate throughout the day, and reduce the burden on the participants. $^{36}$

While a number of studies have investigated EMA for chronic pain, the cyclical nature of menstrual pain implies longer-term monitoring than other chronic pain conditions,

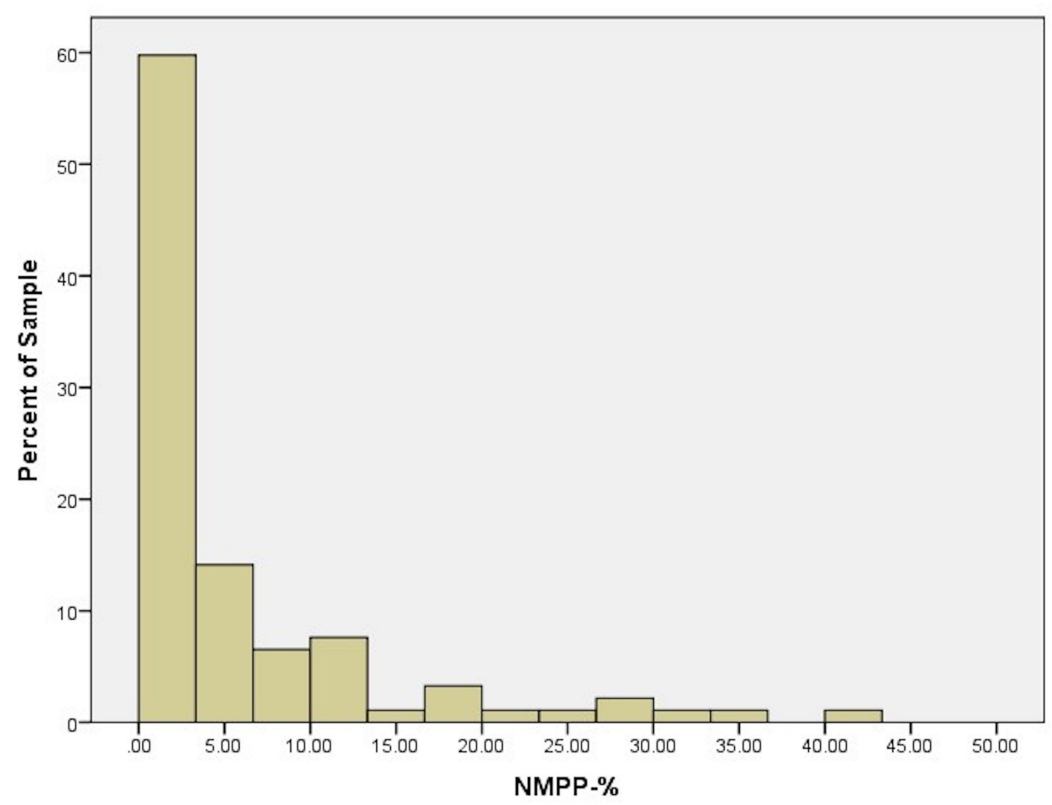

Figure 2 Histogram of NMPP-\% distribution within the whole sample. 

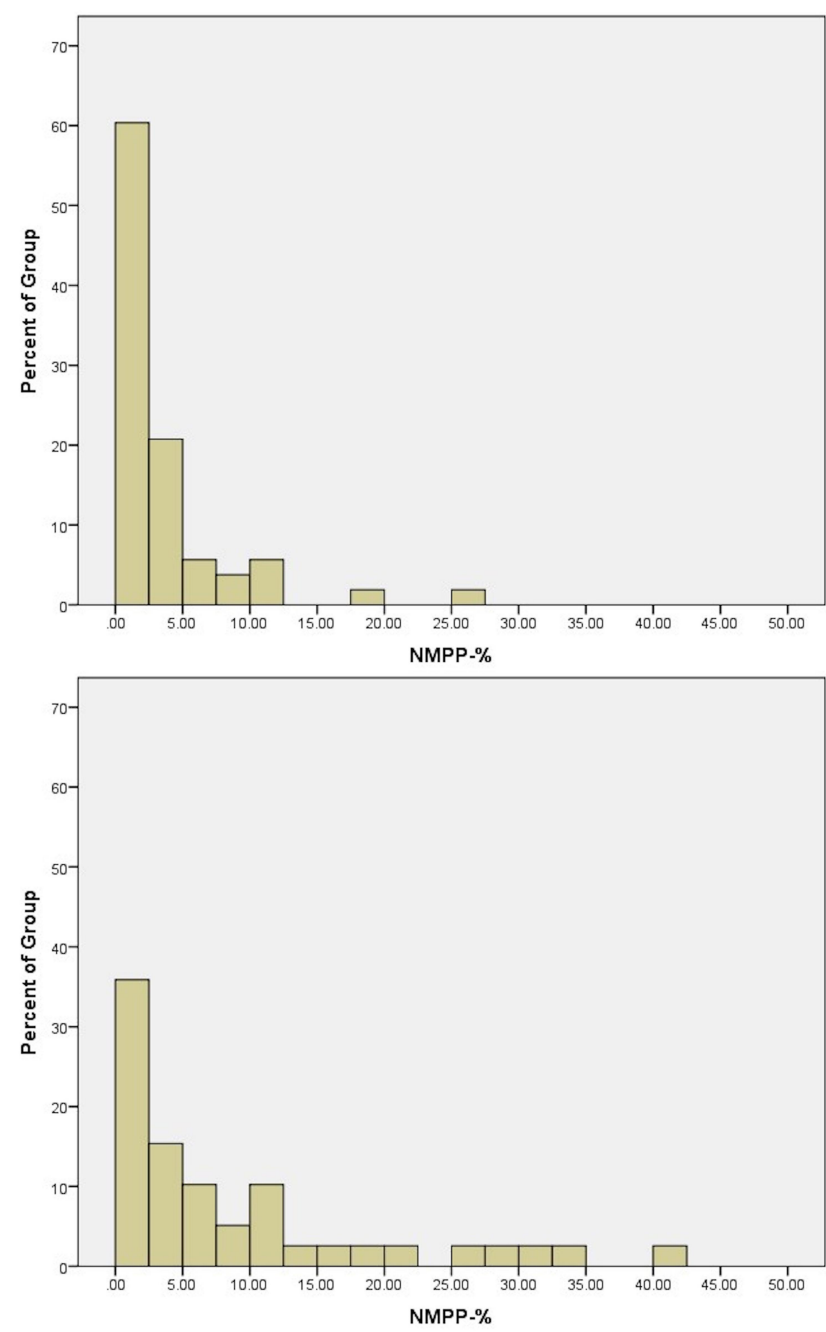

Figure 3 Histograms of NMPP-\% distribution within the control group (A) and PD group (B).

so the needs for EMA in this population might be different than other populations or conditions. Results such as those presented in this manuscript would not be obtained if using the more common shorter EMA duration of 1-2 weeks. ${ }^{17,19}$ These data support the feasibility and acceptability of EMA via EOD text messages for dysmenorrhea research by demonstrating that completion rates remain high over the longer time period, and by showing that a group of otherwise healthy participants with predictable and relatively infrequent pain still find the reporting protocol acceptable.

This is the first known study to demonstrate increased, low-level pelvic pain during non-bleeding days in AYA with $\mathrm{PD}$ as compared to those without PD. Despite the fact that the relatively low intensity and frequency of NMPP observed would generally be considered sub-clinical, these low-level painful experiences may be one mechanism contributing to $\mathrm{CS}$ in AYA with PD. Over time, the persistent nociceptive input experienced during menstruation in women with PD may alter neuronal sensitivity in the central nervous system such that previously non-painful visceral stimuli become painful. ${ }^{4,5,9}$ Given the emergence of this pattern already in girls and younger women, there remain many years of a woman's reproductive lifetime during which this process could unfold.

Limitations to this study must be addressed. The assessments of feasibility and acceptability included in this investigation do not take enrollment rate into consideration. It is possible that some of the individuals who declined participation may have done so because the daily text message procedure was not feasible or acceptable for them. The time of day that the text messages were sent was not identical across study participants. We opted to customize the time of day, within a 4-hour window, to enhance response rate over standardizing the time of day. It is therefore possible that pelvic pain may have occurred after replying to the text message in some participants who chose to receive the texts in the earlier portion of the window. Data were only collected once per day. Typically, EMA protocols for pain assessment sample around five times per day, ${ }^{17,19}$ which enables a more granular examination of the variable of interest and further reduces memory bias. In order to reduce participant burden given the expected length of study participation, we chose to use an EOD protocol, which may have limited our ability to fully capture NMPP experiences.

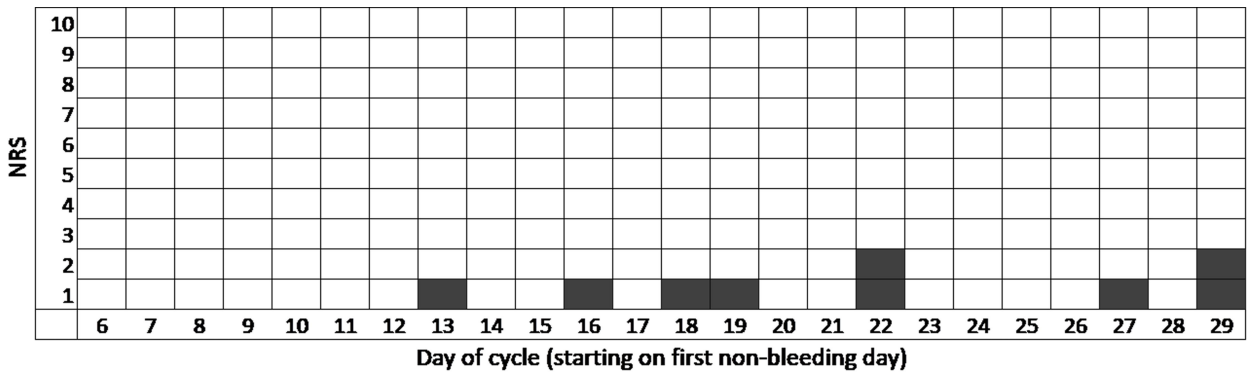

Figure 4 Example of cycle demonstrating low-level pelvic pain across the menstrual cycle. 
The length of each individual's study participation was based on her participation in the larger study and therefore varied; it is possible that standardizing the duration of participation, such as is typical for EMA protocols, may have yielded different results. Studies designed with the primary focus of assessing NMPP in AYA with and without PD are needed to validate the findings presented here. Occasionally participants would answer for the past few days in batches, which may have introduced memory biases. The participant sample from the larger study did not include younger adolescents (eg, 14- to 15-year-olds) and therefore data in this important developmental age are missing from these analyses. The single item pain rating limits other important dimensions of the pain experience, which should be included in future studies of this type. The relatively small sample size limits generalizability. Lastly, despite the extensive screening conducted and the fact that none of the participants met criteria for a diagnosis of chronic pelvic pain, ${ }^{37}$ it is still possible that some participants in the current study may have actually had an underlying organic/structural cause, such as endometriosis or adenomyosis, for their menstrual pain (ie, secondary dysmenorrhea). In this case, the sample would have been heterogenous with regard to the etiology of menstrual pain, which may limit the interpretation of results.

One aspect of this study that could be considered a limitation but that we believe is actually a strength is the interface of text messages. This interface is basic and does not allow for sophisticated data collection, such as would be possible with an app (eg, skip patterning, etc) and does not include graphics, etc. While app-based assessment tools and intervention delivery platforms are extremely popular and growing exponentially, ${ }^{36}$ user fatigue caused by these apps can be high. ${ }^{17}$ A text messagebased approach has limitations to the sophistication of data collection but fits more seamlessly in an individual's life.

\section{Conclusion}

In summary, AYA with PD experience significantly more intense pelvic pain during non-bleeding days and experience pelvic pain more frequently on non-bleeding days than AYA without PD. Though both the intensity and frequency of NMPP are low and at levels that would not typically warrant clinical assessment or intervention, these repeated nociceptive events may represent a potential mechanism that contributes to the transition from cyclical to chronic pelvic pain in some individuals. Additional studies should be conducted to determine the extent to which the observed results can be generalized to other samples of AYA, including those with demographic and socioeconomic characteristics that differ from those of the current sample. Future research is also needed to elucidate the relationships among sub-clinical pelvic pain during non-menstrual cycle phases and potential subsequent clinical implications, including identification of phenotypic subgroups for whom this phenomenon might apply.

\section{Acknowledgments}

This research was supported by National Institutes of Health NICHD grants K23HD077042 (PI: Laura A. Payne), R01HD093680 (PI: Laura A. Payne), and NCATS University of California Los Angeles Clinical and Translational Science Institute grant KL2TR000122 (PI: Laura A. Payne). The content is solely the responsibility of the authors and does not necessarily represent the official views of the NIH.

\section{Disclosure}

Laura Seidman reports grants from NIH, during the conduct of the study. Laura Payne reports grants from NIH, during the conduct of the study. The authors report no other potential conflicts of interest for this work.

\section{References}

1. Iacovides S, Avidon I, Baker FC. What we know about primary dysmenorrhea today: a critical review. Hum Reprod Update. 2015;21 (6):762-778. doi:10.1093/humupd/dmv039

2. Hardi G, Evans S, Craigie M. A possible link between dysmenorrhoea and the development of chronic pelvic pain. Aust $N Z \mathrm{~J}$ Obstet Gynaecol. 2014;54(6):593-596. doi:10.1111/ajo.12274

3. Li R, Li B, Kreher DA, Benjamin AR, Gubbels A, Smith SM. Association between dysmenorrhea and chronic pain: a systematic review and meta-analysis of population-based studies. Am J Obstet Gynecol. 2020;223:350-371. doi:10.1016/j.ajog.2020.03.002

4. Woolf CJ. Central sensitization: implications for the diagnosis and treatment of pain. Pain. 2011;152(3 Suppl):S2-15. doi:10.1016/j. pain.2010.09.030

5. Latremoliere A, Woolf CJ. Central sensitization: a generator of pain hypersensitivity by central neural plasticity. $J$ Pain. 2009;10 (9):895-926. doi:10.1016/j.jpain.2009.06.012

6. Stratton P, Khachikyan I, Sinaii N, Ortiz R, Shah J. Association of chronic pelvic pain and endometriosis with signs of sensitization and myofascial pain. Obstet Gynecol. 2015;125(3):719-728. doi:10.1097/ AOG.0000000000000663

7. Aredo JV, Heyrana KJ, Karp BI, Shah JP, Stratton P. Relating chronic pelvic pain and endometriosis to signs of sensitization and myofascial pain and dysfunction. Semin Reprod Med. 2017;35(1):88-97. doi:10.1055/s-0036-1597123

8. Eller-Smith OC, Nicol AL, Christianson JA. Potential mechanisms underlying centralized pain and emerging therapeutic interventions. Front Cell Neurosci. 2018;12:35.

9. Aslam N, Harrison G, Khan K, et al. Visceral hyperalgesia in chronic pelvic pain. BJOG. 2009;116(12):1551-1555. doi:10.1111/j.14710528.2009.02305.x 
10. Chen T, Mu J, Xue Q, et al. Whole-brain structural magnetic resonance imaging-based classification of primary dysmenorrhea in painfree phase: a machine learning study. Pain. 2019;160(3):734-741. doi:10.1097/j.pain.0000000000001428

11. Liu J, Liu H, Mu J, et al. Altered white matter microarchitecture in the cingulum bundle in women with primary dysmenorrhea: a tract-based analysis study. Hum Brain Mapp. 2017;38 (9):4430-4443. doi:10.1002/hbm.23670

12. Wei SY, Chao HT, Tu CH, et al. Changes in functional connectivity of pain modulatory systems in women with primary dysmenorrhea. Pain. 2016;157(1):92-102. doi:10.1097/j.pain.0000000000000340

13. Payne LA, Rapkin AJ, Seidman LC, Zeltzer LK, Tsao JC. Experimental and procedural pain responses in primary dysmenorrhea: a systematic review. J Pain Res. 2017;10:2233-2246. doi:10.2147/JPR.S143512

14. Payne LA, Seidman LC, Sim MS, Rapkin AJ, Naliboff BD, Zeltzer LK. Experimental evaluation of central pain processes in young women with primary dysmenorrhea. Pain. 2019;160 (6):1421-1430. doi:10.1097/j.pain.0000000000001516

15. Hellman KM, Roth GE, Dillane KE, et al. Dysmenorrhea subtypes exhibit differential quantitative sensory assessment profiles. Pain. 2020;161(6):1227-1236. doi:10.1097/j.pain.0000000000001826

16. Shiffman S, Stone AA, Hufford MR. Ecological momentary assessment. Annu Rev Clin Psychol. 2008;4:1-32. doi:10.1146/ annurev.clinpsy.3.022806.091415

17. May M, Junghaenel DU, Ono M, Stone AA, Schneider S. Ecological momentary assessment methodology in chronic pain research: a systematic review. J Pain. 2018;19(7):699-716. doi:10.1016/j.jpain.2018.01.006

18. Suveg C, Payne M, Thomassin K, Jacob ML. Electronic diaries: a feasible method of assessing emotional experiences in youth? J Psychopathol Behav Assess. 2010;32(1):57-67. doi:10.1007/ s10862-009-9162-0

19. Ono M, Schneider S, Junghaenel DU, Stone AA. What affects the completion of ecological momentary assessments in chronic pain research? An individual patient data meta-analysis. $J$ Med Internet Res. 2019;21(2):e11398. doi:10.2196/11398

20. Vincent K, Warnaby C, Stagg CJ, Moore J, Kennedy S, Tracey I. Dysmenorrhoea is associated with central changes in otherwise healthy women. Pain. 2011;152(9):1966-1975. doi:10.1016/j.pain.2011.03.029

21. Seidman LC, Brennan KM, Rapkin AJ, Payne LA. Rates of anovulation in adolescents and young adults with moderate to severe primary dysmenorrhea and those without primary dysmenorrhea. $J$ Pediatr Adolesc Gynecol. 2018;31(2):94-101. doi:10.1016/j.jpag.2017.09.014

22. Stone AA, Shiffman S. Capturing momentary, self-report data: a proposal for reporting guidelines. Ann Behav Med. 2002;24 (3):236-243. doi:10.1207/S15324796ABM2403_09

23. Wen CKF, Schneider S, Stone AA, Spruijt-Metz D. Compliance with mobile ecological momentary assessment protocols in children and adolescents: a systematic review and meta-analysis. $J$ Med Internet Res. 2017;19(4):e132. doi:10.2196/jmir.6641
24. Breivik EK, Bjornsson GA, Skovlund E. A comparison of pain rating scales by sampling from clinical trial data. Clin J Pain. 2000;16 (1):22-28. doi:10.1097/00002508-200003000-00005

25. Dworkin RH, Turk DC, Farrar JT, et al. Core outcome measures for chronic pain clinical trials: IMMPACT recommendations. Pain. 2005;113(1):9-19. doi:10.1016/j.pain.2004.09.012

26. Castarlenas E, Jensen MP, von Baeyer CL, Miro J. Psychometric properties of the numerical rating scale to assess self-reported pain intensity in children and adolescents: a systematic review. Clin $J$ Pain. 2017;33(4):376-383. doi:10.1097/AJP.0000000000000406

27. von Baeyer CL, Spagrud LJ, McCormick JC, Choo E, Neville K, Connelly MA. Three new datasets supporting use of the numerical rating scale (NRS-11) for children's self-reports of pain intensity. Pain. 2009;143(3):223-227. doi:10.1016/j.pain.2009.03.002

28. Hoenig JM, Heisey DM. The abuse of power: the pervasive fallacy of power calculations for data analysis. Am Stat. 2001;55(1):19-24. doi:10.1198/000313001300339897

29. Levine M, Ensom MH. Post hoc power analysis: an idea whose time has passed? Pharmacotherapy. 2001;21(4):405-409. doi:10.1592/ phco.21.5.405.34503

30. Liao Y, Skelton K, Dunton G, Bruening M. A systematic review of methods and procedures used in ecological momentary assessments of diet and physical activity research in youth: an adapted STROBE checklist for reporting EMA studies (CREMAS). J Med Internet Res. 2016;18(6):e151. doi:10.2196/jmir.4954

31. Hufford MR, Shields AL. Electronic diaries: applications and what works in the field. Appl Clin Trials. 2002;11(4):46-56.

32. Schnall R, Okoniewski A, Tiase V, Low A, Rodriguez M, Kaplan S. Using text messaging to assess adolescents' health information needs: an ecological momentary assessment. J Med Internet Res. 2013;15 (3):e54. doi:10.2196/jmir.2395

33. Pew Research Center. Mobile fact sheet; 2019. Available from: https:// www.pewresearch.org/internet/fact-sheet/mobile/. Accessed July 10, 2020.

34. Schaeffer K. Most U.S. teens who use cellphones do it to pass time, connect with others, learn new things; 2019. Available from: https:// www.pewresearch.org/fact-tank/2019/08/23/most-u-s-teens-who-usecellphones-do-it-to-pass-time-connect-with-others-learn-new-things/. Accessed July 10, 2020.

35. Heimlich R Texting is nearly universal among young adult cell phone owners; 2012; Available from: https://www.pewresearch.org/facttank/2012/12/14/texting-is-nearly-universal-among-young-adult-cellphone-owners/. Accessed July 10, 2020.

36. Hofmann W, Patel PV. SurveySignal: a convenient solution for experience sampling research using participants' own smartphones. Soc Sci Comput Rev. 2014;33(2):235-253. doi:10.1177/0894439314525117

37. Taylor HS, Giudice LC, Lessey BA, et al. Treatment of endometriosis-associated pain with Elagolix, an oral GnRH antagonist. N Engl J Med. 2017;377(1):28-40. doi:10.1056/NEJMoa1700089
Journal of Pain Research

\section{Publish your work in this journal}

The Journal of Pain Research is an international, peer reviewed, open access, online journal that welcomes laboratory and clinical findings in the fields of pain research and the prevention and management of pain. Original research, reviews, symposium reports, hypothesis formation and commentaries are all considered for publication. The manuscript management system is completely online and includes a very quick and fair peer-review system, which is all easy to use. Visit http:// www.dovepress.com/testimonials.php to read real quotes from published authors. 